University of New Orleans

ScholarWorks@UNO

$3-1-2003$

\title{
Spectral polarization measurements by use of the grating division-of-amplitude photopolarimeter
}

Shankar Krishnan

Follow this and additional works at: https://scholarworks.uno.edu/ee_facpubs

Part of the Engineering Commons

\section{Recommended Citation}

Shankar Krishnan, Scott Hampton, James Rix, Brian Taylor, and Rasheed M. A. Azzam, "Spectral Polarization Measurements by Use of the Grating Division-of-Amplitude Photopolarimeter," Appl. Opt. 42, 1216-1227 (2003)

This Article is brought to you for free and open access by the Department of Electrical Engineering at ScholarWorks@UNO. It has been accepted for inclusion in Electrical Engineering Faculty Publications by an authorized administrator of ScholarWorks@UNO. For more information, please contact scholarworks@uno.edu. 


\title{
Spectral polarization measurements by use of the grating division-of-amplitude photopolarimeter
}

\author{
Shankar Krishnan, Scott Hampton, James Rix, Brian Taylor, and \\ Rasheed M. A. Azzam
}

\begin{abstract}
The grating division-of-amplitude photopolarimeter (G-DOAP) is an instrument that exploits the multiple-beam-splitting, polarizing, and dispersive properties of diffraction gratings for the time-resolved measurement of the complete state of polarization of collimated broadband incident light, as represented by the four Stokes parameters as a function of wavelength across the spectrum. It is a compact, high-speed sensor that has no moving parts and is simple to install and operate. These characteristics make the G-DOAP well suited for in situ spectroscopic ellipsometry (SE) applications for monitoring and controlling thin-film processes. The design and performance of a prototype instrument are presented. Precise SE measurements, to $\pm 0.04^{\circ}$ in $\psi$ and $\pm 0.1^{\circ}$ in $\Delta$, are demonstrated in the $550-940$-nm wavelength range. (C) 2003 Optical Society of America
\end{abstract}

OCIS codes: $120.2130,120.6200$.

\section{Introduction}

Four-detector photopolarimeters have certain advantages relative to rotating-analyzer photopolarimeters. They have no moving parts, allowing extremely rapid measurements of the polarization state of light. They are complete polarimeters and yield four Stokes parameters, $S_{0}, S_{1}, S_{2}$, and $S_{3}$, or any four equivalent properties of analyzed light, such as the total intensity, the $p: s$ intensity ratio, the $p: s$ phase difference $\Delta$, and the degree of polarization $P$. Rotating analyzers yield the magnitude but not the sign of $\Delta$, do not yield the degree of polarization, and are less accurate than complete polarimeters in ellipsometric application to very thin films. Furthermore complete polarimeters are required for Mueller-matrix ellipsometry, which yields a complete description of the change in the polarization state of light on reflection from real surfaces.

There are at least three basic types of four-detector photopolarimeters. The four-detector photopola-

When this work was done S. Krishnan, S. Hampton, J. Rix, and B. Taylor were with Containerless Research, Inc., 906 University Place, Evanston, Illinois 60201-3149. S. Krishnan is now at KLATencor, San Jose, California 95134. R. M. A. Azzam is with the Department of Electrical Engineering, University of New Orleans, New Orleans, Louisiana 70148-0001.

Received 17 April 2002; revised manuscript received 6 November 2002 .

0003-6935/03/071216-12\$15.00/0

(C) 2003 Optical Society of America rimeter $^{1}$ (FDP) employs a sequence of four suitably oriented planar detectors that detect an incident beam of defined wavelength and reflect the beam onto the next detector in the sequence. The division-ofamplitude photopolarimeter ${ }^{2-4}$ (DOAP) employs a single phase-shifting beam splitter and two Wollaston (or equivalent) polarizers to deliver monochromatic light to four detectors. Division-of-wave-front devices $^{5}$ are also employed, but they are less accurate than the division-of-amplitude device. The novel technology presented here is based on a polarizationstate sensor known as the grating division-ofamplitude photopolarimeter (G-DOAP). ${ }^{6-9}$ The G-DOAP is based on the multiple-beam-splitting, spectral dispersion, and polarization characteristics of light diffraction by gratings. It yields rapid and complete polarization-state measurements over a wide spectral range.

We describe the operating principles, construction, calibration, and testing of the prototype G-DOAP instrument and the specifications for spectral polarization sensing. The paper is presented in four parts: (1) introduction and technical background, (2) description of the prototype instrument, (3) results of measurements, and (4) discussion of results and future work. Additional information about related DOAP technology for measurements at discrete wavelengths is presented by Krishnan and Nordine. ${ }^{3,10-12}$ ing:

The key results demonstrated here are the follow- 


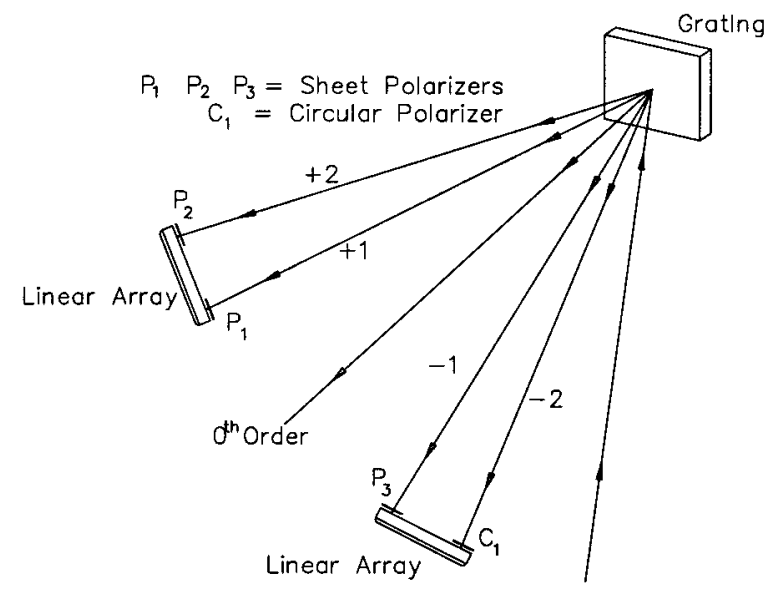

Fig. 1. Schematic drawing of G-DOAP showing the grating, incident and diffracted beams, linear array detectors, and polarizers.

- G-DOAP can measure all four Stokes parameters of arbitrarily polarized light over a 550-940-nm wavelength range.

- Stokes parameters are measured to an absolute accuracy of better than 0.01 over this wavelength range.

- Precision in measuring the azimuth and ellipticity angles is of the order of $0.1^{\circ}$. A spectral resolution of approximately $2-3 \mathrm{~nm}$ has been achieved.

- Full spectral measurements can be obtained at $\sim 2 \mathrm{~Hz}$ (i.e., two full spectropolarimetric scans per second) or better.

- Calibration methods for the G-DOAP are fully developed.

\section{A. Background of the G-DOAP Technology}

The spectral characteristics of grating diffraction are well known and widely exploited in spectroscopy. Light incident on a grating is dispersed into a spectrum, enabling spectroscopy with a slit and grating rotation mechanism, or with a stationary grating and an array detector that intercepts diffracted light. The spectrum is usually measured with the fan-out of only one diffracted order, typically the -1 diffracted order. However, the existence of multiply diffracted and dispersed orders can be exploited to achieve simultaneous spectral and polarization measurements.

The polarization characteristics of the grating diffraction are less known and exploited. In general each of the orders of diffraction contains different information about the polarization state of the light incident on the grating. Therefore, by simultaneously measuring the spectra of four or more orders of diffraction, a grating spectrometer can be designed to yield the complete polarization state for the entire spectrum of light. In practice, polarizing elements are placed in front of two or more detected orders to enhance the sensitivity to the polarized state.

Figure 1 shows the general concept of a G-DOAP instrument. A collimated light beam incident on the grating is dispersed into multiple orders that are detected by multiple linear array detectors. At least 4

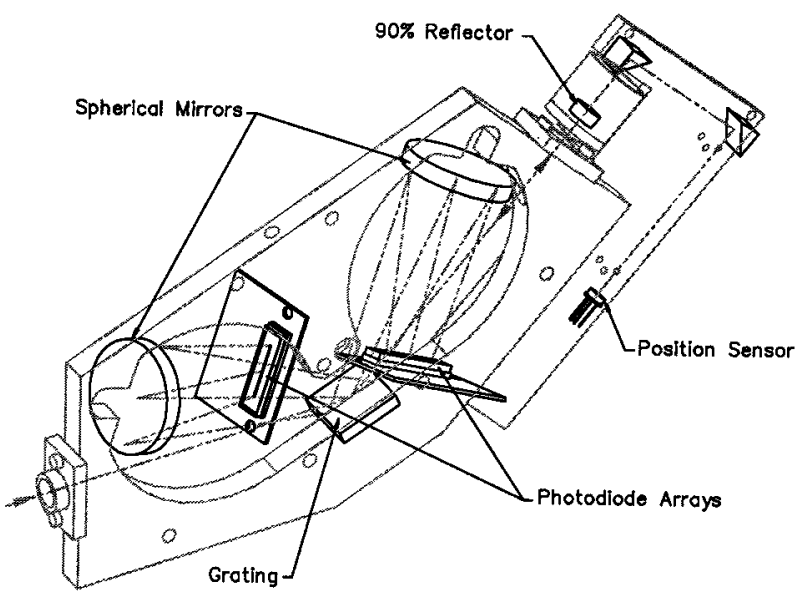

Fig. 2. G-DOAP design implemented in the present research. The first diffraction produces two detected orders while the retroreflected beam produces two additional orders. The location of the arrays with respect to the common plane of incidence is also illustrated.

orders are measured, and at least 2 orders require that polarizers be placed in the beam paths. ${ }^{7}$ The four detected orders provide four independent projections of the unknown Stokes vector of incident light, and measurements of the spectra of the 4 orders allow the incident polarization state to be determined as a function of wavelength. In Fig. 1 we have included four polarizers that are placed in the four diffracted orders. Other requirements for unambiguous detection of the complete polarization state when a G-DOAP is used are described in Ref. 7.

The relationship between the detected intensities and the incident polarization state is determined by a calibration procedure described in Section 3. The diffraction grating disperses light in each order (except the zero order), so that a single grating achieves polarimetric and spectroscopic discrimination of light. Thus the G-DOAP is well suited as a complete polarization-state detector of a spectroscopic ellipsometer. It can also be used as a spectropolarimeter in other applications such as particle-scattering measurements, Mueller-matrix measurements, and generalized ellipsometry.

The design of the prototype G-DOAP instrument described here employs a variation of the concept illustrated in Fig. 1 and is shown in Fig. 2. This design employs a retroreflector that returns the zeroorder beam back to the grating, and the -1 and -2 orders of diffraction are measured for both the incident beam and the retroreflected zero-order beam. Two orders are detected on each array as shown in Fig. 1.

\section{B. G-DOAP in a Spectroscopic Configuration}

Before this work the G-DOAP was not applied to polarization measurements over a continuum of wavelengths. When a collimated, broadband light beam strikes a grating, it is diffracted into multiple orders and dispersed within each order. The diffrac- 
tion efficiency depends on the order number and is a function of wavelength within a given order, and these differences are readily absorbed into the instrument calibration.

The angular characteristics of planar grating diffraction are described by the grating equation

$$
\sin \left(\theta_{i}\right)=\sin (\theta)-i \lambda / g \text {. }
$$

Here $\theta_{i}$ is the angle of diffraction of the $i$ th order, $\theta$ is the angle of incidence (all angles are measured from the grating normal), $\lambda$ is the wavelength of the incident light, and $g$ is the grating period, which is equal to the inverse of the grating spatial frequency.

Although there are several possible schemes by which the G-DOAP concept may be reduced to practice, we have chosen a specific design that accomplishes a compact instrument. The design of this instrument is described in Section 2.

\section{G-DOAP Construction and Characteristics}

\section{A. Overall Design}

Figure 2 shows the G-DOAP design that we developed. The (collimated) incoming beam is incident on an $\mathrm{Al}$-coated, asymmetric sawtooth $\left(3.6^{\circ}\right.$ blaze angle), 150 -groove $/ \mathrm{mm}$ diffraction grating at an incidence angle of $68^{\circ}$. A planar diffraction geometry is used and all diffracted orders are nearly in a common vertical plane. In these conditions the positive orders of diffraction are evanescent and only the zero and negative orders propagate. The first two negative orders $(-1$ and -2$)$ are focused by a spherical mirror onto detector array 1. (A fraction of the -3 order also reaches the array.) The zero-order beam is retroreflected back onto the grating by using a partial (90\%) reflector, and two additional negative orders are directed onto array 2 . The spherical mirrors are slightly tilted so that the two arrays are displaced by $8^{\circ}$ relative to the common plane of diffraction. Approximately $10 \%$ of the light striking the partial reflector is transmitted and focused onto a CCD camera. The image from the CCD camera and the entrance aperture allow precise alignment matching during calibration and measurements.

Four dispersed orders are delivered to the two linear array detectors. Dichroic sheet polarizers of specific orientations are placed directly over the detectors in the different orders. The alignment was performed by illuminating the entrance aperture uniformly while tilting and pointing the instrument such that the beam transmitted by the retroreflector was centered on the position sensor. The mechanical design assured that the return beam was coincident with the first-reflection zero-order beam.

Figure 3 shows the locations of the different dispersed orders and the corresponding pixel-element numbers on a given array. Each array consists of 1024 pixel elements of $25-\mu \mathrm{m}$ width in the dispersion plane and 2.5-mm height. The optical design produces an identical distribution of wavelengths and orders on each array. The locations of the polarizers

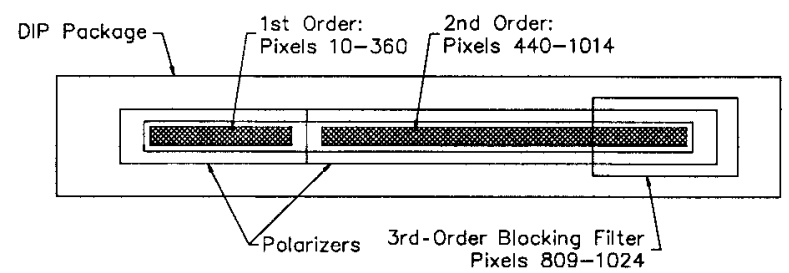

Fig. 3. Location of the first and second diffracted orders on the linear array detectors. The wavelength range is $550-940 \mathrm{~nm}$. The location of the order-blocking filter is also shown (see text).

are also illustrated in Fig. 3. There is an approximate 80-pixel gap between the first and second order to facilitate placement of the polarizers. The gap arises because the spectral range of light entering the G-DOAP is between 550 and $940 \mathrm{~nm}$ and the second diffracted order at $550 \mathrm{~nm}$ begins 80 pixels after the first diffracted order at $940 \mathrm{~nm}$. This also reduces the effect of radiation scattered from the edges of the polarizers. We adjusted the spherical mirror supports to locate the spectra on the arrays by using a reference narrow-band interference filter in the beam path and adjusting the mirrors so that the spectral line was centered at the design pixel locations.

With a minimum design wavelength of $550 \mathrm{~nm}$ the second-order diffraction spectrum at $\lambda=825 \mathrm{~nm}$ begins to overlap with the third-order diffraction beginning at $550 \mathrm{~nm}$. The third-order blocking filter is designed to block the shorter-wavelength third-order light, and its placement is shown in Fig. 3.

\section{B. Pixel-Wavelength Relationship}

Initial experiments with G-DOAP used an analytical relationship between pixel and wavelengths. However, subsequent experiments showed that use of a high-order polynomial function was an excellent way for the relationship between pixels and wavelengths to be described. Accordingly a series of narrow-band interference filters was placed in the light path, and the pixel numbers corresponding to the peak intensity for each case (in each order) were recorded. Typically a set of seven or eight well-characterized filters was used for this wavelength calibration procedure. The center wavelength of each filter was separately characterized with a laboratory spectrometer. A third-order polynomial of the $n=A \lambda^{3}+$ $B \lambda^{2}+C \lambda+D$ type was used, where $n$ is the pixel number; $A, B, C$, and $D$ are the polynomial coefficients determined from regression; and $\lambda$ is the desired wavelength. Interpolation between adjacent pixels was used to account for the fractional pixel value returned from the polynomial function. The polynomial coefficients determined for the four diffracted orders are given in Table 1.

\section{Mechanical Design}

Figure 4 is a combined view of G-DOAP and the polarization-state generator that was used for calibration. A central optical breadboard holds the arrays, grating, and all the optics that make up the G-DOAP instrument. The instrument is supported 
Table 1. Polynomial Coefficients for Wavelength Calibration

\begin{tabular}{ccccc}
\hline $\begin{array}{c}\text { Detector, } \\
\text { Diffraction } \\
\text { Order }\end{array}$ & $A$ & $B$ & $C$ & $D$ \\
\hline $1,-1$ & $-4.334 \times 10^{-7}$ & $7.923 \times 10^{-4}$ & 0.425 & -398.9 \\
$1,-2$ & $-1.007 \times 10^{-6}$ & $1.871 \times 10^{-3}$ & 0.348 & -121.3 \\
$2,-1$ & $-3.278 \times 10^{-7}$ & $5.322 \times 10^{-4}$ & 0.629 & -451.3 \\
$2,-2$ & $-2.907 \times 10^{-7}$ & $2.848 \times 10^{-4}$ & 1.49 & -391.6 \\
\hline
\end{tabular}

by a translation and tilt assembly for alignment purposes. The optical components of the G-DOAP are two array detectors, one grating, two mirrors, a retroreflector, a CCD camera, and two folding mirrors that deliver light to the CCD camera. The optical path length to the CCD detector was long, providing high positioning sensitivity owing to the long lever arm. In the calibration mode a fraction of the light flux incident on the G-DOAP sensor was reflected at a small angle $\left(<5^{\circ}\right)$ by a beam splitter onto a reference detector. The output of the reference detector was used to normalize the array outputs during calibration.

The dimensions of the G-DOAP prototype sensor are as follows: length, 8 in. $(20.3 \mathrm{~cm})$ width, 6 in. $(14.7 \mathrm{~cm})$; and height, 6 in. $(14.7 \mathrm{~cm})$. Two timing cables, two video cables, and one signal cable are routed from the sensor to the digital signal processing (DSP) module.

\section{Component Selection for the G-DOAP System}

The G-DOAP system consists of the light source and calibration optics as well as the G-DOAP sensor and its electronics.

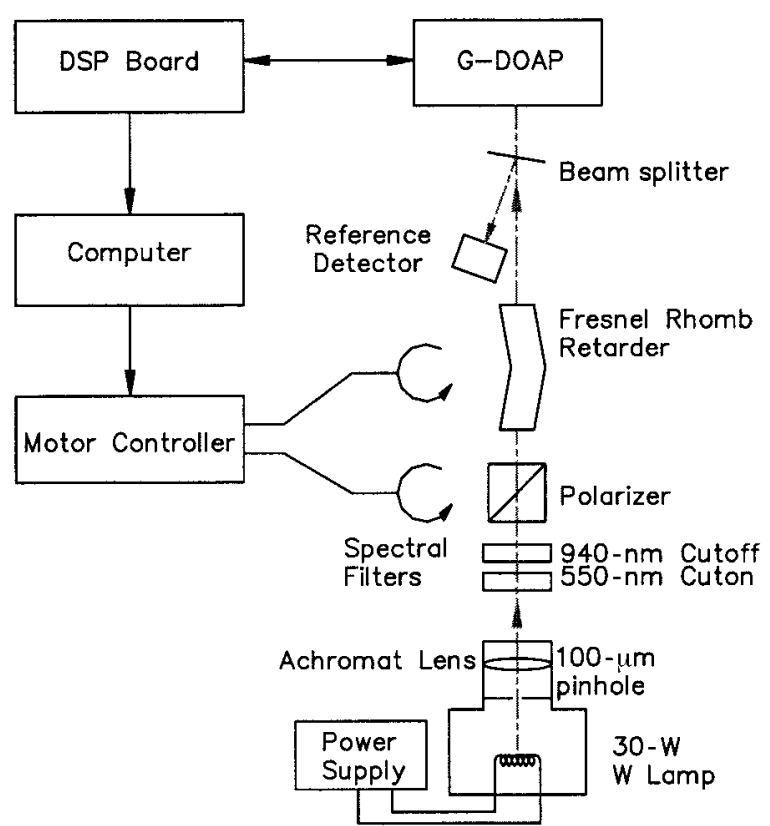

Fig. 4. Schematic drawing of the system used for G-DOAP calibration, verification, and ellipsometric measurements.

\section{Light Source and Calibration Optics}

All measurements were obtained with a 20-W tungsten-filament lamp. Arc-lamp sources were considered and were not used because of the large variation in intensity over the spectrum. Collimating optics achieved a beam divergence of $\sim 0.8 \mathrm{mrad}$. The dispersion plane of the grating is vertical. In these conditions the G-DOAP received sufficient light to use integration times of $\sim 100 \mathrm{~ms}$ for data readout from the array detectors. A larger horizontal divergence can be used within the G-DOAP without affecting the spectral resolution.

A Glan-Thomson crystal polarizer with very low beam deviation was used as the linear polarizer. The polarizer and the quarter-wave retarder (see below) were mounted in $0.001^{\circ}$ precision rotation stages.

The most stringent requirement for the G-DOAP calibration is the use of a quarter-wave retarder (QWR) to measure the last column of the instrument calibration matrix F (see Section 3). It has been shown ${ }^{13}$ that an ideal QWR is not needed to achieve an accurately calibrated polarimeter system. However, there are only a few achromatic QWR designs commercially available that operate over the entire wavelength range of interest.

The Fresnel rhomb is the most achromatic of all QWR designs. For the present work a fourreflection BK7 Fresnel rhomb from Halbo Optics was used. Although the rhomb does have some dispersion, the variation in retardance over the wavelength range of interest was sufficiently small that it could be used to calibrate the G-DOAP. However, rotation of the rhomb did cause some beam motion.

\section{G-DOAP Components}

Two different gratings with 150 grooves $/ \mathrm{mm}$ were tested. A (sinusoidal-profile) holographic grating was found to be unsuitable owing to a strong Wood's anomaly ${ }^{14}$ in the 560-640-nm region. A ruled and blazed grating was found to exhibit a less conspicuous Wood's anomaly. All the results presented here were obtained by using the ruled and blazed grating (away from the Littrow condition). Ruled and blazed gratings are common and inexpensive but generate faint ghost diffraction spectra owing to small variations in the groove spacing and can also yield greater intensity in high diffraction orders than the holographic gratings.

The linear detector arrays were two Hamamatsu 1024-element, $25-\mu \mathrm{m}$ pitch, 2.5-mm-high $n$-type metal-oxide semiconductor (NMOS) self-scanning photodiode arrays. These are the most expensive components of the G-DOAP.

Corning Polarcor glass polarizers were used. The average spectral transmittance and extinction ratio of these polarizers are shown in Fig. 5. The rapid reduction in transmission and extinction are evident at the ends of the spectral region.

Two position sensors were used. The first was a quadrant detector. Later a CCD camera was em- 


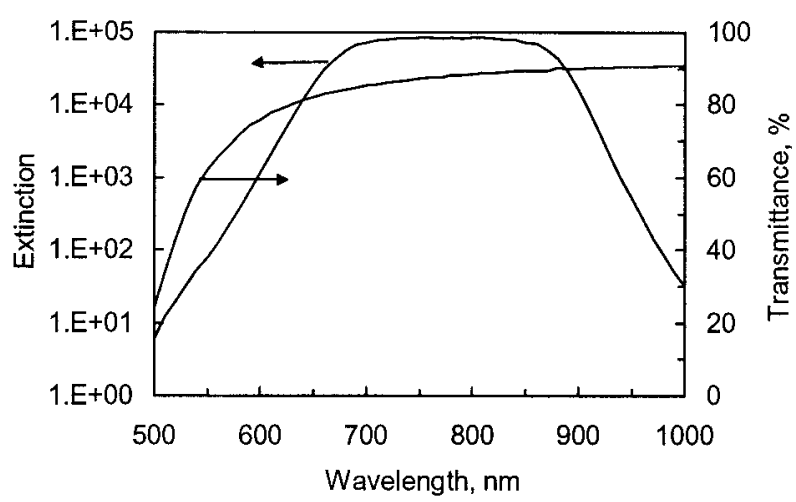

Fig. 5. Transmittance (right axis) and extinction of the Polarcor (TM) sheet polarizers used in the G-DOAP.

ployed. The CCD camera provides a full image of the beam with good sensitivity for use in position detection and alignment.

\section{E. Electronics, Timing, and Data Acquisition}

\section{Electronics and Timing}

A DSP-based platform was used to perform array timing, data acquisition, and data transfer. The DSP system was designed and fabricated by Indus Instruments, Houston, Tex. A schematic block diagram of the various components of the DSP system is shown in Fig. 6. The DSP generates timing pulses to drive both the arrays and the master clock, start and stop pulses, and all the clocks required by the arrays.

The DSP board included 16-bit analog-to-digital converters (ADCs) for the array outputs and 12-bit ADCs for the outputs of the quadrant detector and a reference detector used during calibration. Digital outputs from the DSP board operated a mechanical shutter (placed in the light path) for background subtraction. The DSP board included sufficient onboard memory for temporary storage of as many as 100 scans of the array outputs.

The DSP allowed variable integration times and on-board averaging of the data. The integration times and averaging could be set dynamically from the host PC. For all the measurements reported here an integration time of $100 \mathrm{~ms}$ was used for three of the orders and an integration time of $300 \mathrm{~ms}$ was

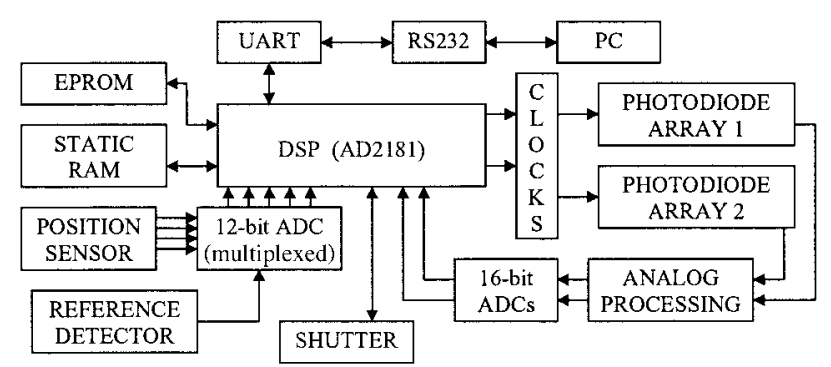

Fig. 6. Schematic block diagram of the electronics system showing the ADCs, DSP, and serial communications: EPROM, erasable, programmable ROM; UART, universal asynchronous receiver transmitter. used for the other order. The integration times were cycled in rapid succession.

Future operation of the DSP could include a lookup table to convert the pixel position into a wavelength, storage of the instrument matrix at each wavelength, and direct conversion of the data into Stokes and ellipsometric parameters. These facilities would eliminate the need for a host PC to operate the system.

\section{Data Acquisition}

A serial communications scheme was used in the present work. Data transfer over the serial bus occurred in $\sim 1 \mathrm{~s}$. Incorporation of a Universal Serial Bus interface would reduce the data-transfer period to less than $20 \mathrm{~ms}$.

Data were displayed in real time on a PC operating in Windows 98. A custom program was used for calibration, alignment, measurements, and other tests.

\section{F. Typical Spectra}

The spectroscopic polarization data from the G-DOAP consist of the two array detector outputs. Each array has 1024 pixels shared by two dispersed orders of diffraction, thus providing four intensity measurements at each wavelength. The dispersion in the second order is twice that of the first. The spectral resolution of the G-DOAP was limited by the divergence of the light beam incident on the grating to $\sim 2 \mathrm{~nm}$. The dispersion in the first order was slightly greater than $1 \mathrm{~nm} /$ pixel and smaller than the spectral resolution.

Our convention refers to the first two orders of diffraction generated by the incoming light beam and detected at array 1 as orders 1 and 2 and the first two orders generated by the retroreflected light beam and detected at array 2 as orders 3 and 4 .

Figure 7 shows spectra obtained with the G-DOAP for incident light, which is linearly polarized parallel and perpendicular to the plane of diffraction as well as incident right circularly polarized light. The four different orders of diffraction are identified in each part of Fig. 7. The Wood's anomaly occurs in the neighborhood of $625 \mathrm{~nm}$ and is most pronounced in the parallel polarization response, as expected. Note that the decrease in diffraction efficiency occurs in two of the four orders; fortunately neither order has zero or near-zero efficiency in the region of the anomaly.

Spectral resolution of the order of 2-3 nm was verified by locating the pixel positions of the different diffracted orders with incident monochromatic light at 633-, 685-, and 900-nm wavelengths obtained by inserting calibrated narrow-band interference filters in the light path.

\section{G. Choice of Polarizer Azimuths}

There are numerous orientations of the sheet polarizers that may be used in G-DOAP, but there are only a few orientations that provide a robust (nonsingular) instrument matrix and high accuracy in measurements of the polarization state at all wavelengths. The choice of polarizer orientation was narrowed by 

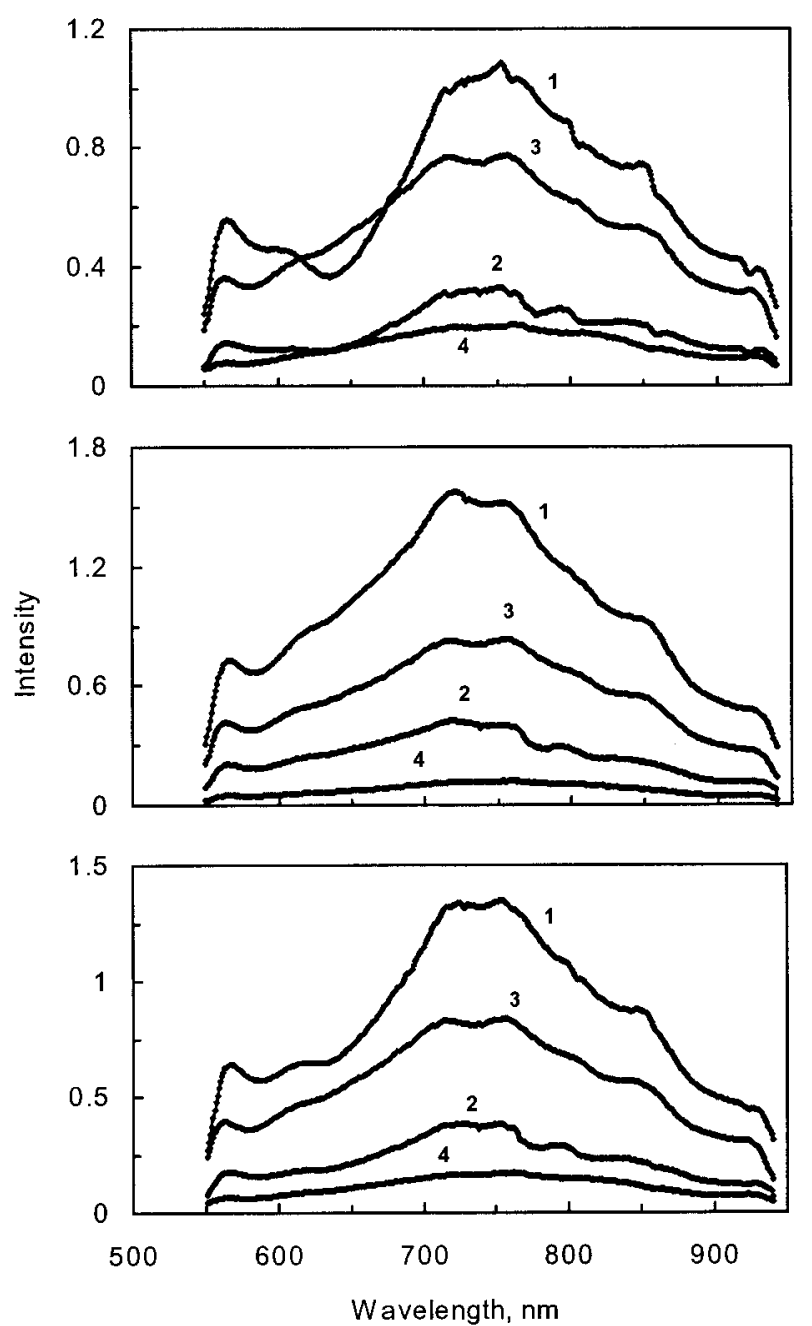

Fig. 7. Intensity of the four diffracted orders versus wavelength for top, incident parallel $p$ polarization; middle, perpendicular $s$ polarization; and, bottom, right-circular polarization.

two independent methods. First, the Mueller matrix of each order was measured with a Muellermatrix ellipsometer at $532 \mathrm{~nm}$, and the instrument matrix was numerically computed and optimized for different polarizer orientations. The second approach involved inspecting the results after the instrument response was measured without any polarizers and then making educated guesses. For all the research presented here the G-DOAP employed polarizer orientations of $90^{\circ}$ in order $1,45^{\circ}$ in order $2,-45^{\circ}$ in order 3 (first-order diffraction onto the second array detector), and $0^{\circ}$ in order 4 (secondorder diffraction onto the second array detector). A birefringent retarder sheet (140 $\mathrm{nm}$ of retardance) was placed over the polarizer for order 2 .

\section{G-DOAP Calibration, Verification, and Measurements}

A. Principles of Polarization Measurements with the G-DOAP

Light enters the G-DOAP as a beam reflected by a sample in an ellipsometer configuration or straight through from a polarization-state generator during calibration, as shown in Fig. 4. The polarization state of this light is characterized by the Stokes vector $\mathbf{S}=\left[S_{0} S_{1} S_{2} S_{3}\right]^{t}$, where $t$ stands for the transpose. The response of the G-DOAP is given by four voltages (intensities), $\mathbf{I}=\left[\begin{array}{llll}I_{0} & I_{1} & I_{2} & I_{3}\end{array}\right]^{t}$ measured by the four detectors on four orders of diffraction for a given wavelength. The intensities corresponding to a given wavelength from each of the orders yields the intensity matrix I, which is related to the input Stokes vector $\mathbf{S}$ through the instrument matrix $\mathbf{F}$ :

$$
\mathbf{I}=\mathbf{F S},
$$

where $\mathbf{F}$ is a $4 \times 4$ real matrix that is a characteristic of the instrument. If $\mathbf{F}$ is known, $\mathbf{S}$ can be calculated from I by using the inverse relationship:

$$
\mathbf{S}=\mathbf{F}^{-1} \mathbf{I} \text {. }
$$

Once $\mathbf{F}$ is known as a function of wavelength, the G-DOAP can be used as an analyzer in a spectroscopic ellipsometer. The measured Stokes parameters are related to the ellipsometric parameters through simple trigonometric functions that depend on the choice of incident polarization state.

Any photopolarimeter, including the G-DOAP, measures the polarization state of light that is characterized by the four-element Stokes vector, consisting of the four Stokes parameters $S_{0}, S_{1}, S_{2}, S_{3}$. In practice, the normalized Stokes parameters (NSPs) $S_{1} / S_{0}, S_{2} / S_{0}$, and $S_{3} / S_{0}$ are sufficient to describe the polarization state.

\section{B. Calibration Procedure}

Calibration of the G-DOAP is achieved by determining the $4 \times 4$ real matrix $\mathbf{F}$ at each wavelength of interest. The calibration setup is shown in Fig. 4. The calibration is completed by a two-step procedure. ${ }^{13}$

During the first step of a calibration the retarder is removed and the polarizer is rotated through $360^{\circ}$ of azimuth. The first part of the calibration determines the first three columns $\mathbf{F}_{0}, \mathbf{F}_{1}$, and $\mathbf{F}_{2}$ of the instrument matrix $\mathbf{F}$ by inputting linear polarization states of azimuths ranging from $0^{\circ}$ to $360^{\circ}$. The response of the G-DOAP can be written as ${ }^{13}$

$$
\mathbf{I}(P)=\mathbf{F}_{0}+\mathbf{F}_{1} \cos (2 P)+\mathbf{F}_{2} \sin (2 P) .
$$

In Eq. (4) $\mathbf{I}(P)$ is the four-element vector of normalized intensities for the four orders of diffraction. $\mathbf{F}_{0}$, $\mathbf{F}_{1}$, and $\mathbf{F}_{2}$ are determined by a simple Fourier-type least-squares fit to Eq. (4) of the voltages (normalized by the reference detector voltage) obtained for each order intercepted by the G-DOAP.

The second step of the calibration determines the fourth column $\mathbf{F}_{3}$ by measurement of the response of the G-DOAP to right- and left-circular polarization states. The Fresnel rhomb QWR is inserted into the optical path and rotated to $+45^{\circ}$ and $-45^{\circ}$ relative to the polarizer transmission axis to produce these states. The Stokes vectors for right- and leftcircular states are $\left[\begin{array}{llll}1 & 0 & 0 & 1\end{array}\right]^{\mathrm{t}}$ and $\left[\begin{array}{llll}1 & 0 & 0 & -1\end{array}\right]^{\mathrm{t}}$, re- 


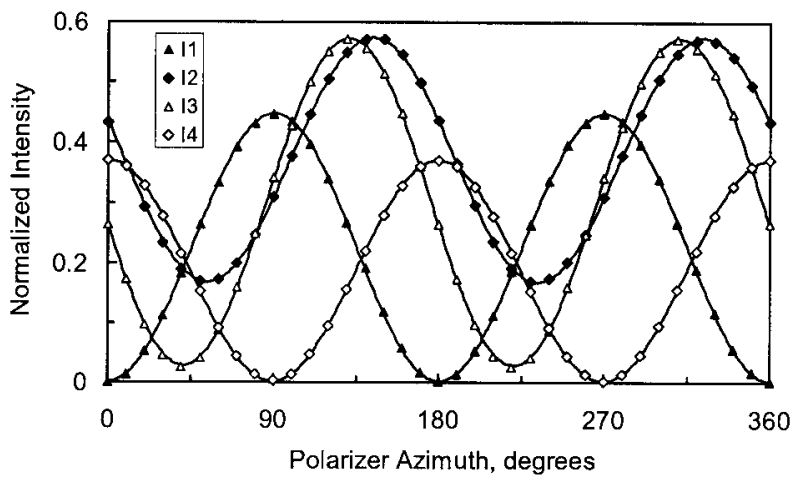

Fig. 8. Detector responses, normalized by the reference detector intensity, for the four diffracted orders versus the polarizer azimuth for a $700-\mathrm{nm}$ wavelength: symbols, measured points in $20^{\circ}$ polarizer steps; curves, least-squares fits to the data described by Eq. (4).

spectively. The corresponding relationships for obtaining $\mathbf{F}_{3}$ are

$$
\mathbf{I}(\mathrm{RCP})=\mathbf{F}_{0}+\mathbf{F}_{3}, \quad \mathbf{I}(\mathrm{LCP})=\mathbf{F}_{0}-\mathbf{F}_{3},
$$

where RCP and LCP refer to the right- and leftcircular polarization states, respectively. Therefore $\mathbf{F}_{3}$ can be obtained from

$$
\mathbf{F}_{3}=0.5[\mathbf{I}(\mathrm{RCP})-\mathbf{I}(\mathrm{LCP})] .
$$

A separate consistency check on $\mathbf{F}_{0}$, the first column of $\mathbf{F}$, is also obtained:

$$
\mathbf{F}_{0}=0.5[\mathbf{I}(\mathrm{RCP})+\mathbf{I}(\mathrm{LCP})] .
$$

$\mathbf{F}_{0}$ obtained from Eq. (7) should agree with that obtained from Eq. (4). In practice a multiplicity of nominally right- and left-circular states is generated by rotating the polarizer and retarder together to several optically equivalent positions and averaging the results. A total of 18 right- and left-nearlycircular states was used in the current work. The averaged results were used to compute $\mathbf{F}_{3}$ from Eq. (6).

Figure 8 shows typical results obtained in the first step of calibration for a wavelength of $700 \mathrm{~nm}$. The symbols correspond to the measured intensities (normalized by the reference detector intensity) of the four individual orders versus the polarizer azimuth. The solid curves represent the least-squares fits to the curves represented by Eq. (4). The coefficients of the fits make up the first three columns of the instrument matrix. Equation (7) was then used as a consistency between the results obtained with linear and circular states. In practice the two measurements of $\mathbf{F}_{0}$ always agreed within $2 \%$ or better over the entire wavelength range.

To evaluate the performance of G-DOAP as a func-

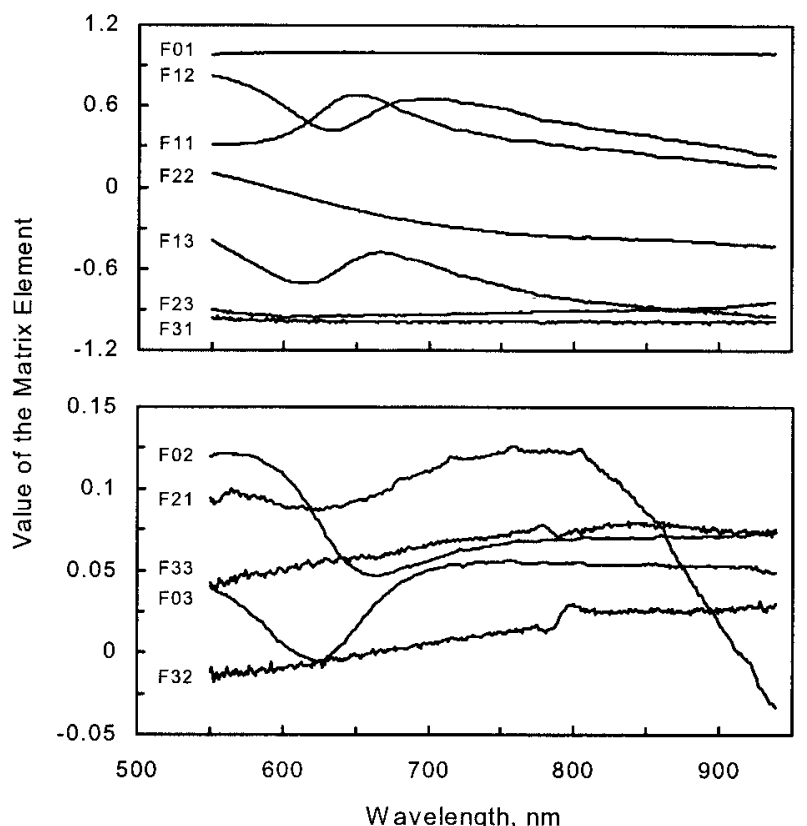

Fig. 9. Elements of the normalized calibration matrix $\mathbf{F}_{n}$ versus wavelength for the G-DOAP. Note the strong dispersion in the vicinity of $625 \mathrm{~nm}$, caused by the Wood's anomaly.

tion of wavelength, the $\mathbf{F}$ matrix is normalized according to

$$
\mathbf{F}_{n}=\left[\begin{array}{llll}
1 & F_{01} & F_{02} & F_{03} \\
1 & F_{11} & F_{12} & F_{13} \\
1 & F_{21} & F_{22} & F_{23} \\
1 & F_{31} & F_{32} & F_{33}
\end{array}\right] .
$$

In Fig. 9 is plotted the elements in the second, third, and fourth columns versus wavelength over the range of interest. The reduced extinction ratio of the polarizers at both ends of the spectral range causes corresponding changes in the elements near 550 and $940 \mathrm{~nm}$. Wood's anomaly ${ }^{14}$ contributes to the variations near the $625-\mathrm{nm}$ wavelength. A smooth variation in the calibration matrix elements with wavelength is observed over the entire spectral region.

One measure of the polarimetric sensitivity of G-DOAP is given by the absolute values of the four normalized projection vectors ${ }^{15}$ (NPVs) whose components are the nonunit elements in the four rows of the normalized matrix $\mathbf{F}_{n}$. Figure 10 shows the spectral dependence of the NPV magnitudes. The deviations of those magnitudes from unity are caused by the nonideal behavior of the dichroic polarizers and increase at the short- and long-wavelength ends of the spectral range.

The overall quality of the calibration matrix of the G-DOAP is assessed by computing the determinant of the normalized matrix $\mathbf{F}_{n}$. The determinant of this matrix determines the extent to which deviations in the intensity measurements couple into deviations in the derived Stokes parameters. Larger determinants produce smaller errors in the Stokes parame- 


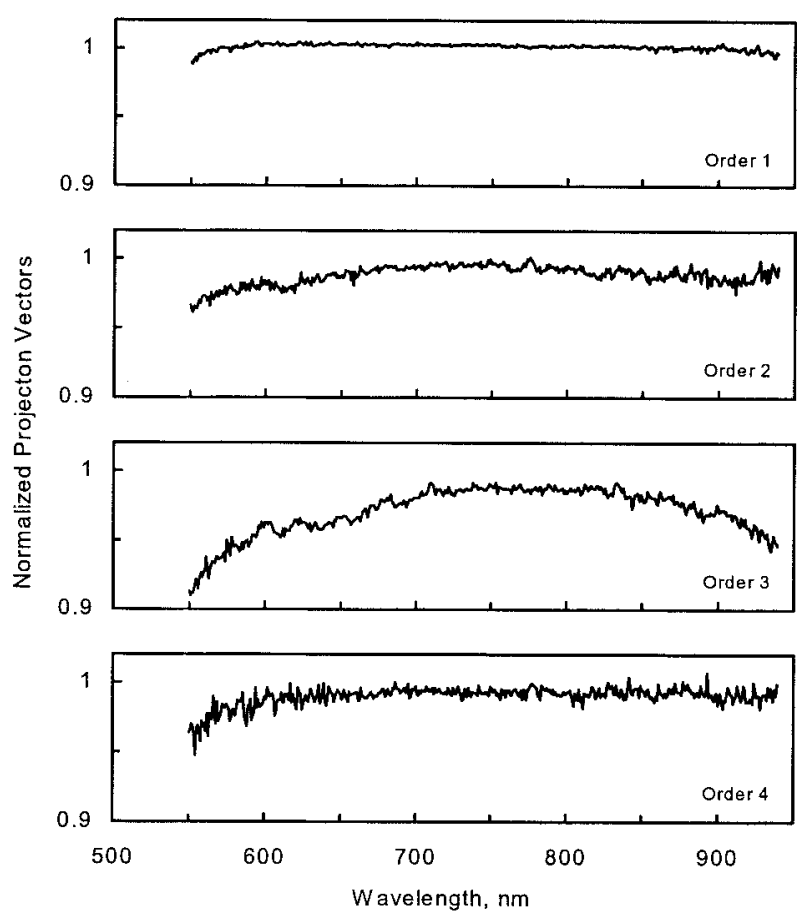

Fig. 10. Magnitudes of the NPV's of the G-DOAP.

ters. ${ }^{4}$ Figure 11 presents the determinant as a function of wavelength. These results suggest how the precision of measurements can be improved by changes in the instrument design. The decrease in the determinant near 550 and $940 \mathrm{~nm}$ is a direct result of the limited extinction ratio of the Polarcor polarizers at those wavelengths. The dip in the determinant around $650 \mathrm{~nm}$ is related to the Wood's anomaly and may be diminished by an appropriate grating design.

Note that the theoretical upper limit on the magnitude of the determinant is equal to $16 / 3^{1.5}=3.0792$ (see the derivation in Appendix A). The maximum value of the experimental determinant in Fig. 11 falls short of this theoretical limit by a factor of 2 . This result indicates that there is further room for optimization of the G-DOAP design.

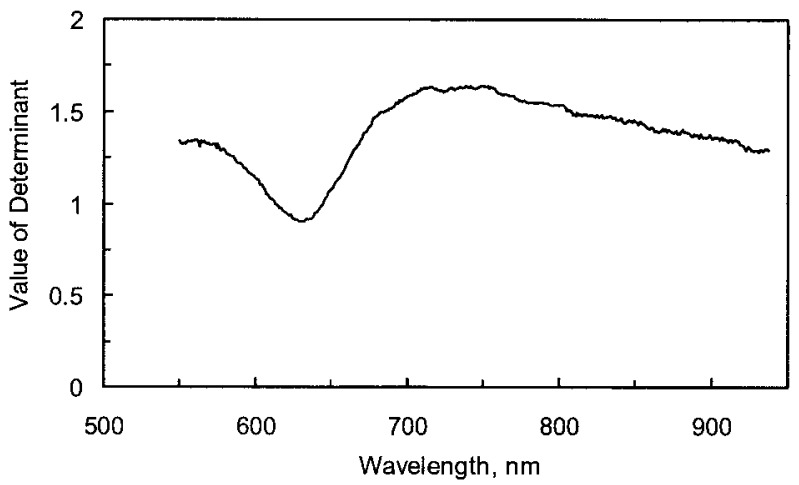

Fig. 11. Determinant of the matrix $\mathbf{F}_{n}$ as a function of wavelength. The reduced magnitude of the determinant near $625 \mathrm{~nm}$ is due to Wood's anomaly.

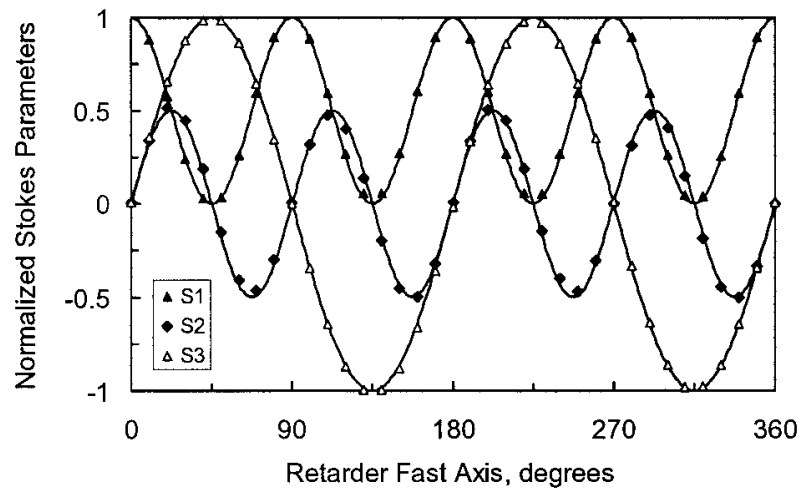

Fig. 12. Measured values of the normalized Stokes parameters (NSP) versus the retarder fast-axis azimuth for the rotating- $C$ test at $700 \mathrm{~nm}$ : symbols, measured points in $10^{\circ}$ fast-axis steps; curves, predictions for an ideal linear retarder.

\section{G-DOAP Verification}

The ultimate verification of the performance of the G-DOAP will be assessed by integrating the system in a spectroscopic ellipsometer and using it for realtime process control applications. Here we describe verification of the G-DOAP performance by using the calibration matrices obtained at each wavelength to measure laboratory-generated polarization states. The Fresnel-rhomb retarder was rotated through $360^{\circ}$ to produce a continuum of linear, circular, and elliptical states from the linearly polarized state transmitted by the Glan-Thompson polarizer. When the retarder fast axis $C$ rotates through $360^{\circ}$, with the linear polarizer fixed at the $P=0$ azimuth, the resultant NSPs are given by

$$
\begin{aligned}
& S_{1}=0.5+0.5 \cos (4 C) \\
& S_{2}=0.5 \sin (4 C) \\
& S_{3}=\sin (2 C)
\end{aligned}
$$

The procedure was as follows. First, the outputs of the linear array detectors were measured over the given wavelength range as a function of the retarder fast-axis azimuth. Then the calibration matrices obtained above were inverted, and Eq. (3) was used to compute the Stokes parameters. The results were then compared with the polarization states determined by Eqs. (9).

Representative results from the rotating- $C$ test at $700 \mathrm{~nm}$ are shown in Fig. 12. The solid symbols are the NSPs, $S_{1}, S_{2}$, and $S_{3}$, measured by the G-DOAP for retarder fast-axis azimuth steps of $10^{\circ}$. The curves are values predicted by Eqs. (9) for an ideal linear QWR. Clearly the data are close to the predicted values but some departures are observed.

Figure 13 shows deviations of the NSPs from the predicted values for the same $700-\mathrm{nm}$ wavelength. Since the retarder azimuths of $C$ and $C+180^{\circ}$ are equivalent, one expects the deviations at these azimuths to be identical apart from scatter in the measurements. This is nearly true, although slight noncyclic trends with azimuth are also seen. 


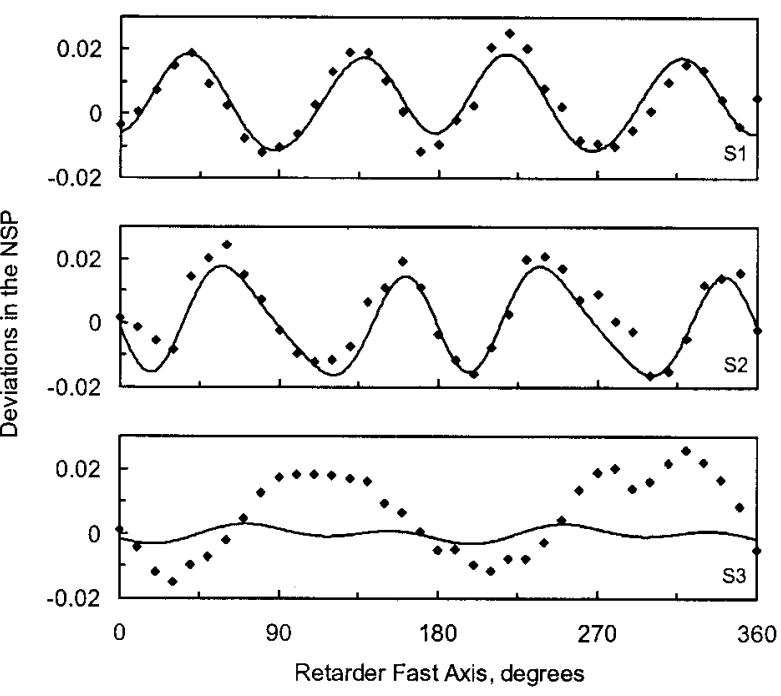

Fig. 13. Deviations of the NSP versus the retarder fast-axis azimuth for the rotating- $C$ test at $700 \mathrm{~nm}$ from values predicted for an ideal linear retarder. Note the asymmetry in the deviations across the two $180^{\circ}$ segments that are due to imperfections in the retarder. The solid curves are fits to the deviations when the model for the retarder described by Azzam and Lopez ${ }^{13}$ is used.

The systematic variation of the deviations results mostly from imperfections in the retarder (in particular, deviation of the retardance from $90^{\circ}$ ) and also from beam-deviation errors. These deviations can be fitted with the imperfect-QWR model of Azzam and Lopez. ${ }^{13}$ The solid curves in Fig. 13 are derived from the model applied to the present data. It is apparent from Fig. 13 that fits to $\Delta S_{1}$ and $\Delta S_{2}$ are quite good while fits to $\Delta S_{3}$ are not as good. This arises because the model ${ }^{13}$ for $\Delta S_{3}$ does not have as many parameters as for $\Delta S_{1}$ or $\Delta S_{2}$ and does not contain a dc term.

The model also provides a measure of the retardance of the QWR. The actual retardance that we obtain with this model is plotted versus the wavelength in Fig. 14 and is compared with the calculated dispersion of retardance of the BK7 Fresnel rhomb, which is shown by the dashed line. Although the

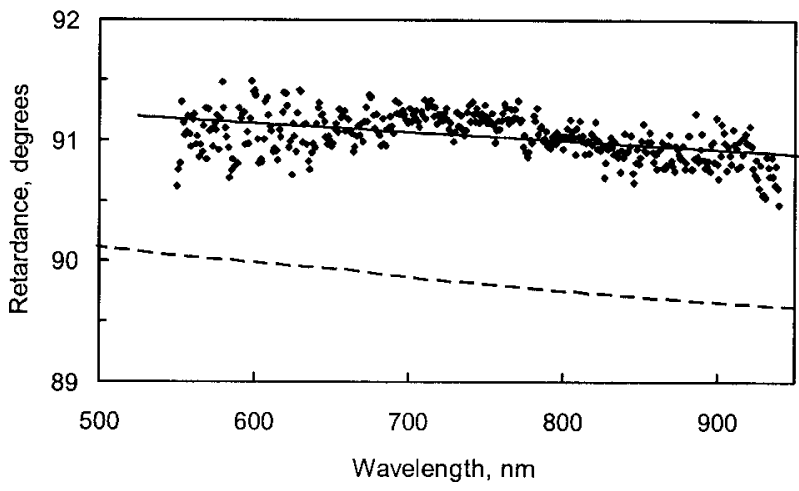

Fig. 14. Calculated retardance as a function of wavelength derived from the model and comparison with the calculated dispersion curve for, dashed line, the BK7 Fresnel rhomb. Solid line, a linear fit, included for clarity.

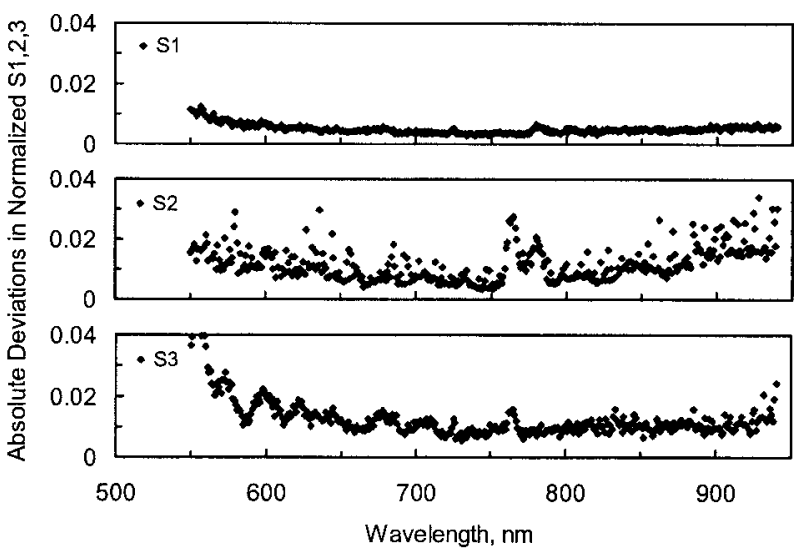

Fig. 15. Average deviation of the NSP over a wavelength range of $550-940 \mathrm{~nm}$. The deviations are the residual errors that remain between the model fit and the data in Fig. 13.

model retardance shows the general trend of falling off as the wavelength is increased, there is a systematic upward shift in the estimated retardance value of $\sim 1.2^{\circ}$ and some fluctuations of retardance with a wavelength of the order of $\pm 0.3^{\circ}$. A line showing a linear fit to the experimental data is included for clarity.

Figure 15 shows the average absolute value of the deviations of the NSPs as a function of wavelength. The data in Fig. 15 were obtained by summing the absolute values of the residual deviations of the data from the model fit (see Fig. 13) for each wavelength and taking the average value. If one excludes the short- and long-wavelength ends of the spectrum, it is apparent that most of the deviations fall below 0.01 . There are also some higher deviations near $775 \mathrm{~nm}$ caused by light scattering from the edge of the orderblocking filter.

\section{Spectroscopic Ellipsometry Measurements}

If the input polarizer is fixed at $+45^{\circ}$ with respect to the plane of incidence in an ellipsometer, the ellipsometric parameters $\psi$ and $\Delta$ are unambiguously obtained (including the signs) if all four Stokes parameters of light reflected from the sample surface are measured. The ellipsometric parameters are then given by

$$
\begin{aligned}
& \Psi=\frac{1}{2} \tan ^{-1}\left[\frac{\left(S_{3}^{2}+S_{2}^{2}\right)^{1 / 2}}{-S_{1}}\right], \\
& \Delta=\tan ^{-1}\left[\frac{-S_{3}}{S_{2}}\right] .
\end{aligned}
$$

Figure 16 shows the ellipsometric spectra of $\psi$ and $\Delta$ as obtained by spectroscopic ellipsometry that employs G-DOAP as the polarization-state detector from an oxidized $\mathrm{Si}$ wafer $\left(\mathrm{a} \mathrm{SiO}_{2}\right.$ layer thickness of $\sim 913.7$ $\mathrm{nm})$ at a $75^{\circ}$ angle of incidence. The least-squares fit to the data in Fig. 16 yields a film thickness of 913.5 nm. 


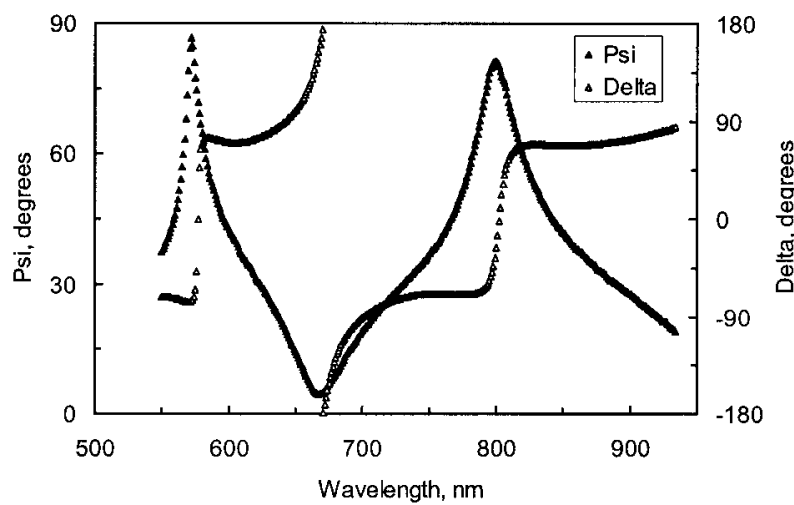

Fig. 16. Measured values of the ellipsometric parameters $\psi$ and $\Delta$ as a function of wavelength for a thick $\mathrm{SiO}_{2}$ film on $\mathrm{Si}$ at an angle of incidence of $75^{\circ}$. The derived value of the film thickness was $913.5 \mathrm{~nm}$ compared with $913.7 \mathrm{~nm}$ obtained with a commercial spectroscopic ellipsometer.

\section{E. Precision Measurements}

To assess the precision of G-DOAP in the measurement of the Stokes parameters and consequently of $\psi$ and $\Delta$, a series of experiments was performed with laboratory-generated polarization states. The precision is determined primarily by the precision of the intensity measurements. The data-acquisition system was set to acquire 50 sequential measurements at high rates and with no signal averaging. The intensities were recorded and normalized for each wavelength by the intensities recorded in the first set of measurements.

Figure 17 gives the distribution of the NSP values at a $700-\mathrm{nm}$ wavelength for the case in which the
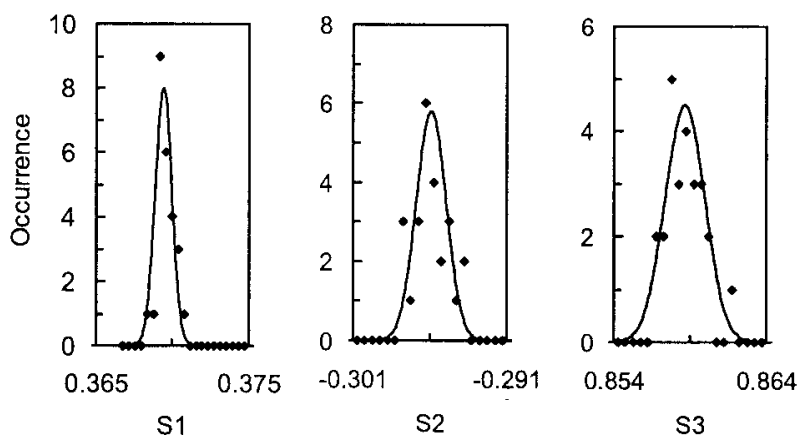

Fig. 17. Distribution of the NSP values derived from a series of 50 measurements for a 700-nm wavelength. signal levels on the arrays were optimal. From these results we estimate the standard deviation to be of the order of $0.0006,0.0010$, and 0.0013 in $S_{1}, S_{2}$, and $S_{3}$, respectively. The measured Stokes parameters were used to extract the values of $\psi$ and $\Delta$. To estimate the precision in $\psi$ and $\Delta$, two separate experiments were performed in which the overall light intensity was varied by adjusting the light intensity. Two cases were analyzed:

Case 1: Signal level on order 2 was $90 \%$ of full scale; signal levels on orders 1,3 , and 4 were at $\sim 20 \%$ of full scale.

Case 2: Signal levels were $45 \%$ of the signal levels in case 1 .

Measurement of the NSP requires measurement of four different orders on two different arrays to extract the Stokes parameters. Thus the noise characteristics of each array contribute differently to the overall precision. The noise on array 2 was somewhat larger than the noise on array 1 . The approximate noise standard deviation on array 2 was $\pm 7 \mathrm{mV}$, while it was $\pm 5 \mathrm{mV}$ on array 1 . Taking the smallest signal on array 2 and correspondingly the largest noise value, we can compute an approximate (or effective) electronic signal-to-noise $(\mathrm{S} / \mathrm{N})$ ratio and compare it with the approximate standard deviation of $\psi$ and $\Delta$ for the two cases of large signal (case 1 ) and small signal (case 2).

The results are in Table 2, assuming that the precision is determined purely by photometric precision and furthermore that the smallest signal on array 2 dominates the precision. While this assumption will not be strictly accurate, there is a more refined method to assess the precision of the data, the analysis of Brudzewski. ${ }^{4}$ We have chosen not to employ this method here since only an estimate is needed.

The second case in Table 2 is not a realistic measurement situation, since three out of the four signals were less than $10 \%$ of full scale and the maximum $\mathrm{S} / \mathrm{N}$ (electrical) is only 100 . We believe that the noise floor can be reduced by factors of $2-3$ so that $\psi$ and $\Delta$ precision better than $\pm 0.05^{\circ}$ will be possible over the entire wavelength range. Signal averaging (not used in the present work) could also be employed while a data rate of one to two full spectroscopic ellipsometry measurements per second are maintained. In these conditions the G-DOAP offers a

Table 2. Relationship between Electrical $\mathrm{S} / \mathrm{N}$ and $\psi$ and $\Delta$ Precision

\begin{tabular}{|c|c|c|c|c|c|c|}
\hline \multirow[b]{2}{*}{$\begin{array}{l}\text { Wavelength, } \\
(\mathrm{nm})\end{array}$} & \multicolumn{3}{|c|}{ Case 1, Higher Light Level } & \multicolumn{3}{|c|}{ Case 2, Lower Light Level } \\
\hline & $\begin{array}{l}\text { Electronic } \\
\mathrm{S} / \mathrm{N}\end{array}$ & $\begin{array}{c}\text { Precision } \\
\psi\end{array}$ & $\begin{array}{c}\text { Degree } \\
\Delta\end{array}$ & $\begin{array}{l}\text { Electronic } \\
\mathrm{S} / \mathrm{N}\end{array}$ & $\begin{array}{c}\text { Precision } \\
\psi\end{array}$ & $\begin{array}{c}\text { Degree } \\
\Delta\end{array}$ \\
\hline 550 & 65 & 0.16 & 0.23 & 25 & 0.37 & 0.4 \\
\hline 600 & 95 & 0.06 & 0.18 & 40 & 0.1 & 0.3 \\
\hline 700 & 270 & 0.03 & 0.06 & 95 & 0.06 & 0.1 \\
\hline 800 & 170 & 0.03 & 0.047 & 100 & 0.04 & 0.12 \\
\hline 900 & 130 & 0.06 & 0.33 & 70 & 0.1 & 0.6 \\
\hline
\end{tabular}


precision in $\psi$ and $\Delta$ measurements of $0.1^{\circ}$ or better over the entire wavelength range.

Table 2 shows that the $\mathrm{S} / \mathrm{N}$ values change by a larger amount at smaller wavelengths when the total intensity is reduced. This difference resulted from the method used to reduce intensity; i.e., the lamp power was reduced so that the intensity changed by a greater fraction at smaller wavelengths than at larger wavelengths.

The standard deviations in $\psi$ and $\Delta$ increase near the ends of the spectrum. The deviations increase because (1) the signal levels decrease and (2) the determinant of the instrument matrix decreases. The precision can also be increased by reducing the electrical noise in the detectors. The 7-10-mV noise represents a maximum electrical S/N of only 1000:1. The sources of the noise are discussed in Section 4.

In summary the G-DOAP has a reproducibility of $\psi$ and $\Delta$ measurements of the order of $0.1^{\circ}$. The precision (and the absolute accuracy) in $\psi$ and $\Delta$ are independent of the value of $\psi$ or $\Delta$, and the precision is determined purely by photometric considerations.

\section{Discussion}

\section{A. Accuracy}

The accuracy of the G-DOAP is determined by the accuracy with which it is calibrated, and this involves measurements with rotating polarizers and retarders. Since the retarders contain imperfections, the accuracy cannot be established by assessing the deviations in the rotating- $C$ test, although it is known ${ }^{13}$ that a perfect retarder is not necessary to obtain an accurate calibration. Independent assessment of the G-DOAP accuracy will be obtained by taking measurements on well-characterized reference samples.

Given that the crystal linear polarizer used in calibration is nearly ideal, the deviations relative to the predicted values for the NSPs may be used to estimate an absolute accuracy over the wavelength range. Based on an average deviation of 0.005 in the NSP over the wavelength range of interest, we estimate that the absolute accuracy of the G-DOAP based on polarimetric measurements alone is $\sim 0.1^{\circ}$ in $\psi$ and $\Delta$.

\section{B. Precision}

The results from a series of precision measurements show that the repeatability of G-DOAP measurements of $\psi$ and $\Delta$ values for a relatively low $\mathrm{S} / \mathrm{N}$ (in the range of $100-200)$ is $\pm 0.1-0.2^{\circ}$. The precision can be improved by signal averaging, by using a brighter light source, increasing the light collection efficiency, and reducing detector noise. At present the integration times were $\sim 400 \mathrm{~ms}$ (total), and this would permit a total data rate of approximately two complete spectroscopic ellipsometric measurements per second. If the overall light level were increased by a factor of 4 , the precision could be increased by a factor of 2 by averaging the signal and keeping the data throughput the same.
The measurements were done with a beam of rather small divergence from a relatively lowbrightness light source. We can also achieve increased intensities without reducing the spectral resolution by increasing the length of the slit at the light source. This change would increase the horizontal divergence of the beam received by the G-DOAP but would not change the divergence in the plane of diffraction.

\section{Sources of Noise}

Three sources of noise were identified: broadband noise from the array boards, clock noise, and detector dark current noise. Our data show the difference in the noise level of one array board compared with the other in spite of the two boards having identical gains in the optical-amplifier stages. We estimate that the overall background noise level can be reduced to $\sim 3 \mathrm{mV}$ (a reduction of a factor of 2 ). This reduction would permit electrical $\mathrm{S} / \mathrm{N}$ values of 300:1 for signals at $10 \%$ of full scale, a typical minimum signal that may be encountered in real measurement circumstances.

\section{Order Blocking}

Figure 3 showed the layout of the various orders on the detector arrays and the order-blocking filter that prevents overlap between the -3 order in the $550-$ $627-\mathrm{nm}$ region with the -2 order of the 825-940-nm range. One problem yet to be overcome is the scattering of light at the edge of the blocking filter. This scattering gives rise to a deterioration in performance in the vicinity of $775 \mathrm{~nm}$ (Fig. 15).

\section{E. Control of Light Source}

In calibrating G-DOAP the intensity measurements were normalized by the total intensity received by the instrument. This was accomplished by the reference detector, as illustrated in Fig. 4. There is one additional requirement that was not controlled in the calibration, i.e., that the lamp spectrum not change during the calibration measurements. Although a highly regulated lamp power supply was used, it was not demonstrated that the lamp output was constant. Changes in the lamp output would result from changes in the filament temperature, and these changes would be larger at small wavelengths than at large wavelengths. Therefore normalization of the G-DOAP measurements by the total intensity is not strictly correct.

\section{F. Future Research}

In the present paper we have provided a detailed description of the design and performance of the G-DOAP instrument. It will be followed by papers on application of this instrument to ellipsometric measurements on thin and thick oxide films on silicon wafers and in Mueller-matrix ellipsometry. The research has identified design issues for further development of this instrument technology, which include (1) changes to the mirror assembly to increase the precision with which the spectra are located and fo- 
cused on the array detectors, (2) improvements of the DSP code to permit real-time integration switching, (3) PC board level improvements to the array electronics to reduce broadband electronic noise, (4) evaluation of higher brightness light sources, (5) evaluation of trade-offs between size, spectral resolution, and the precision and accuracy of polarizationstate measurements, and (6) the use of multiple gratings in a design strategy that would allow measurements only in first order and eliminate the need for order-blocking filters.

\section{Appendix A: Maximum Determinant of the Normalized Instrument Matrix}

In this appendix we determine the upper limit on the determinant of the normalized instrument matrix of G-DOAP and other four-detector photopolarimeters. To simplify notation, the components of the four normalized projection vectors are written in the form $\left(x_{i}\right.$, $y_{i}, z_{i}$ ), where $i=0,1,2$, and 3 . Hence the determinant is given by

$$
D=\left|\begin{array}{llll}
1 & x_{0} & y_{0} & z_{0} \\
1 & x_{1} & y_{1} & z_{1} \\
1 & x_{2} & y_{2} & z_{2} \\
1 & x_{3} & y_{3} & z_{3}
\end{array}\right|
$$

When the elements of the first row from the corresponding elements of the remaining rows are subtracted, the determinant is unchanged and is reduced in size to

$$
D=\left|\begin{array}{lll}
x_{1}-x_{0} & y_{1}-y_{0} & z_{1}-z_{0} \\
x_{2}-x_{0} & y_{2}-y_{0} & z_{2}-z_{0} \\
x_{3}-x_{0} & y_{3}-y_{0} & z_{3}-z_{0}
\end{array}\right| .
$$

The determinant in Eq. (A1) can be written as a triple scalar product of three vectors:

$$
D=\mathbf{A} \cdot(\mathbf{B} \times \mathbf{C}),
$$

where the components of the three vectors, $\mathbf{A}, \mathbf{B}$, and $\mathbf{C}$, are the elements of the three rows of the determinant of Eq. (A1). Thus the magnitude of the determinant equals the volume of the parallelepiped whose three neighboring sides are $\mathbf{A}, \mathbf{B}$, and $\mathbf{C}$ or six times the volume of the pyramid with these same sides. With the ideal polarizers in front of the detectors in all diffracted orders, the vertices of this pyramid are on the unit-radius Poincaré sphere. Consequently the maximum determinant corresponds to six times the maximum-volume pyramid circumscribed by the unit sphere. It is simple to show that the latter volume equals $8 / 3^{2.5}$, so that the maximum possible determinant is given by

$$
D_{\max }=\frac{16}{3^{1.5}}=3.0792 .
$$

This work was supported in part by a National Science Foundation (NSF) Phase I and Phase II Small Business Innovative Research (SBIR) award. Special thanks are due to Paul Nordine for help with the manuscript and to S. Madala of Indus Instruments for help with the DSP electronics.

\section{References}

1. R. M. A. Azzam, E. Masetti, I. M. Elminyawi, and F. B. Grosz, "Construction, calibration, and testing of a four-detector photopolarimeter," Rev. Sci. Instrum. 59, 84-88 (1988).

2. R. M. A. Azzam, "Division-of-amplitude photopolarimeter (DOAP) for the simultaneous measurement of all four Stokes parameters of light," Opt. Acta 29, 685-689 (1982).

3. S. Krishnan, "Calibration, properties, and applications of the division-of-amplitude photopolarimeter at 632.8 and 1523 nm," J. Opt. Soc. Am. A 9, 1615-1622 (1992).

4. K. Brudzewski, "Static Stokes ellipsometer: general analysis and optimization,” J. Mod. Opt. 38, 889-896 (1991).

5. E. Collett, "Determination of the ellipsometric characteristics of optical surfaces using nanosecond laser pulses," Surf. Sci. 96, 156-167 (1980).

6. R. M. A. Azzam, "Division-of-amplitude photopolarimeter based on conical diffraction of light from a metallic grating," Appl. Opt. 31, 3574-3576 (1992).

7. R. M. A. Azzam and K. A. Giardina, "Photopolarimeter based on planar grating diffraction," J. Opt. Soc. Am. A 10, 11901196 (1993).

8. M. A. Azzam, "Diffraction grating photopolarimeters and spectrophotopolarimeters," U.S. Patent 5,337,146 (9 August 1994).

9. Y. Cui and R. M. A. Azzam, "Sixteen-beam grating-based division-of-amplitude photopolarimeter," Opt. Lett. 21, 89-91 (1996).

10. S. Krishnan and P. C. Nordine, "Mueller-matrix ellipsometry using the division of amplitude photopolarimeter: a study of depolarization effects," Appl. Opt. 33, 4184-4192 (1994).

11. S. Krishnan and P. C. Nordine, "Fast ellipsometry and Mueller-matrix ellipsometry using the division-of-amplitude photopolarimeter," in Polarization Analysis and Applications to Device Technology, T. Yoshizawa and H. Yokota, eds., Proc. SPIE 2873, 152-156 (1996).

12. S. Krishnan, "Mueller-matrix ellipsometry on electroformed, rough surfaces," J. Mod. Opt. 42, 1695-1706 (1995).

13. R. M. A. Azzam and A. G. Lopez, "Accurate calibration of the four-detector photopolarimeter with imperfect polarizing optical elements," J. Opt. Soc. Am. A 6, 1513-1521 (1989).

14. See, e.g., C. H. Palmer and F. W. Phelps, "Grating anomalies as local phenomenon,” J. Opt. Soc. Am. 58, 1184-1188 (1968).

15. R. M. A. Azzam, "Instrument matrix of the four-detector photopolarimeter: physical meaning of its rows and columns and constraints on its elements," J. Opt. Soc. Am. A 7, 87-91 (1990). 